\title{
Avaliação de qualidade do pão tipo francês por métodos instrumentais e sensoriais
}

\author{
Evaluation of quality of bread type french by instrumental and \\ sensory methods
}

\author{
Larissa Raphaela Gonçalves de Farias Feitosa ${ }^{1 *}$; Janeeyre Ferreira Maciel²; $^{2}$, \\ Tainá Amaral Barreto ${ }^{3}$; Ricardo Targino Moreira ${ }^{2}$
}

\begin{abstract}
Resumo
$\mathrm{Na}$ presente pesquisa, o objetivo foi avaliar a qualidade do pão tipo francês, comercializado em panificadoras de João Pessoa-PB, por meio de métodos instrumentais e sensoriais. Inicialmente, amostras de pães das doze panificadoras selecionadas nesse estudo foram submetidas às determinações de volume específico, umidade, $\mathrm{pH}$, acidez, cor da casca, cor do miolo e perfil de textura, tendo todas essas análises sido realizadas em triplicata, com 10 repetições. Em seguida, foi aplicado um teste de aceitação sensorial, utilizando-se uma escala hedônica de nove pontos, com 65 provadores, que avaliaram as amostras quanto aos atributos aparência, cor da casca, cor do miolo, crocância da casca, aroma, sabor, textura e aceitação global. Nos testes instrumentais, foi observado que a maioria dos pães apresentou umidade abaixo de $26 \%$ e acidez acima de $4,5 \mathrm{~mL}$ de $\mathrm{NaOH}$ 0,1N/10 g de pão. Com relação ao volume específico, valores satisfatórios foram observados em $86,2 \%$ das amostras (entre 5,0 e 8,0 $\mathrm{cm}^{3} / \mathrm{g}$ ), enquanto para a luminosidade da casca (60-78), e firmeza $(2,87$ a $6,32 \mathrm{~N})$, esse percentual foi em torno de $80 \%$. Para as demais características de textura foi observado que a média da elasticidade variou de $0,91-1,93$, da coesividade de $0,72-0,86$ e da mastigabilidade de 1,91 -5,71 N. Em relação à aceitação sensorial, somente $50 \%$ das panificadoras tiveram seus pães aceitos em todos os atributos avaliados, com escores médios iguais ou superiores a 6 . As demais amostras foram rejeitadas por falta de crocância, e uma destas teve seus pães rejeitados também nos atributos aroma, sabor e aparência, apresentando, portanto, maior necessidade de adequação.
\end{abstract}

Palavras-chave: Pão, parâmetros de qualidade, aceitação sensorial

\begin{abstract}
The objective of the study was to evaluate the quality of French type bread from bakeries of João Pessoa - PB - Brazil using sensory and instrumental methods. Initially, samples from twelve bakeries selected in this study were submitted for determination of specific volume, moisture, $\mathrm{pH}$, acidity, crust color, crumb color and texture profile, all of these analyses have been performed in triplicate, with 10 repetitions. A sensory acceptance test was applied using a 9-point hedonic scale, with 65 tasters. The samples were evaluated considering attributes of appearance, crust color, crumb color, crust crispness, aroma, taste, texture and the overall acceptability. In instrumental tests, it was observed that most of samples showed moisture below $26 \%$ and acidity above $4.5 \mathrm{~mL}$ of N/10 NaOH $0.1 \mathrm{~N} / 10 \mathrm{~g}$ of bread. With respect to the specific volume, satisfactory values were observed in $86,2 \%$ of samples (between 5.0 and $8.0 \mathrm{~cm}^{3} / \mathrm{g}$ ), while for skin brightness $(60-78)$ and firmness $(2.87$ to $6.32 \mathrm{~N})$, this percentage was
\end{abstract}

${ }^{1}$ Eng $^{\mathrm{a}}$ de Alimentos, Mestre em Ciência e Tecnologia de Alimentos, Universidade Federal da Paraíba, UFPB, João Pessoa, PB. E-mail: larissargfarias@gmail.com

2 Engenheiros de Alimentos, Profs. Drs., UFPB, João Pessoa, PB. E-mail: janeeyre@ct.ufpb.br; ricardo.ufpb@gmail.com

3 Discente em Engenharia de Alimentos, UFPB, João Pessoa, PB. E-mail: tainaamaral_alimentos@hotmail.com

* Autor para correspondência 
around $80 \%$. In other textural characteristics was observed that the elasticity varied from $0.91-1.93$, cohesiveness $0.72-0.86$ and chewiness of $1.91-5.71 \mathrm{~N}$. In relation to sensory acceptance, only $50 \%$ of the bakeries have had their bread accepted in all evaluated attributes, with average scores similar or superior to 6 . The other samples were rejected due to the lack of crispness, and one of those also had its bread rejected in the attributes of aroma, taste and appearance, therefore showing increased need for adequacy.

Key words: Bread, quality parameters, sensory acceptance

\section{Introdução}

O pão francês é o tipo de pão mais consumido no Brasil, sendo considerado uma das principais fontes calóricas da dieta dos brasileiros (IBGE, 2010). É obtido basicamente por mistura de farinha de trigo, cloreto de sódio, fermento e água, podendo ser adicionado de alguns aditivos como enzimas, agentes oxidantes e hidrocolóides, entre outros (BÁRCENAS; O-KELLER; ROSELL, 2009; JOYE; LAGRAIN; DELCOUR, 2009) e é caracterizado por apresentar casca crocante, de cor uniforme castanho-dourada e miolo de cor brancocreme, de textura e granulação fina não uniforme. Entretanto, mudanças na formulação e processo de elaboração podem causar variações nessas características, resultando na obtenção de produtos com qualidade variável.

Algumas das medidas que contribuem para a redução da variabilidade na produção do pão francês são padronização de formulação, uso de câmaras de fermentação e de controle de temperatura do forno, condições que dependem de investimentos em equipamentos, manutenção e capacitação de pessoal (GIANNOU; KESSOGLOU; TZIA, 2003).

Com maior uniformidade na produção, é possível iniciar um processo de estabelecimento de padrões de qualidade para o pão francês, que deverão ser monitorados por meio de análises instrumentais e sensoriais. As principais características avaliadas por métodos instrumentais são volume específico, umidade, firmeza, cor e porosidade do miolo e cor da casca (ESTELLER; LANNES, 2005; CURIC et al., 2008). Com relação aos testes sensoriais, os mais aplicados são análise descritiva e teste de aceitação (HAYAKAWA et al., 2010, CALLEJO, 2011).
Considerando a crescente exigência dos consumidores, o controle da variabilidade na produção e, consequentemente, a melhoria da qualidade do pão francês passa a ser uma prioridade para panificadoras que têm o intuito de manter e atrair mais clientes.

Nesta pesquisa, o objetivo foi avaliar a qualidade do pão tipo francês, produzido em pequenas panificadoras de João Pessoa-PB, por meio de métodos instrumentais e sensoriais, adotando como critérios de qualidade a uniformidade, atendimento a padrões pré-estabelecidos e a satisfação do consumidor.

\section{Material e Métodos}

\section{Material}

Amostras de pão francês, produzidas em doze panificadoras de João Pessoa-PB, foram obtidas mediante compra. As panificadoras foram selecionadas por meio de sorteio, a partir de 120 estabelecimentos cadastrados no Sindicato dos Panificadores do Estado da Paraíba - SINDIPAN/ $\mathrm{PB}$, sendo codificadas por letras, variando de $\mathrm{A}$ a $\mathrm{M}$.

\section{Coleta das amostras}

Para as análises instrumentais, três unidades de pão francês foram obtidas por coleta, sendo este procedimento repetido 10 vezes, durante duas semanas, com exceção dos sábados e domingos, totalizando 30 unidades de pão, por panificadora. Considerando que nesse intervalo de tempo amostras de duas panificadoras eram coletadas, as coletas das 12 panificadoras ocorreram em 12 
semanas, entre os meses de março e junho de 2012. Para a avaliação sensorial, foi realizada somente uma coleta de 65 unidades de pão francês, o que ocorreu em diferentes dias para cada panificadora, durante os meses de julho e agosto de 2012. Todas as amostras foram coletadas durante a manhã e transportadas até o laboratório em sacos de papel.

Avaliação dos parâmetros de qualidade do pão francês

Amostras de pão francês das doze panificadoras selecionadas tiveram sua qualidade avaliada por meio de análises instrumentais e sensoriais.

\section{Análises instrumentais}

Foram determinados volume específico, umidade, cor da casca e do miolo, $\mathrm{pH}$, acidez e perfil de textura.

O volume específico foi determinado pelo método de deslocamento de sementes de painço, calculandose o resultado pela razão entre o volume $\left(\mathrm{cm}^{3}\right)$ e massa do pão $(\mathrm{g})$, sendo expresso $\mathrm{em} \mathrm{cm}^{3} / \mathrm{g}$ (AACC 10-11, 2000). O teor de umidade foi analisado em estufa a $105^{\circ} \mathrm{C}$ até peso constante (AOAC, 1997). Para $\mathrm{pH}$ e acidez, homogeneizou-se 10 gramas de cada amostra com $90 \mathrm{~mL}$ de água destilada, e o pH da suspensão resultante foi determinado utilizando potenciômetro modelo 0400 (Quimis, São Paulo, Brasil), previamente calibrado e operado de acordo com as instruções do fabricante. Em seguida, a suspensão foi titulada com solução de $\mathrm{NaOH} 0,1 \mathrm{~N}$ até $\mathrm{pH}$ 8,5. A acidez titulável foi expressa em $\mathrm{mL}$ de $\mathrm{NaOH} 0,1 \mathrm{~N}$ consumido por $10 \mathrm{~g}$ de pão (ROBERT et al., 2006).

A análise de cor do pão francês foi realizada em três diferentes pontos na crosta e três no miolo, sendo os resultados de cada parte expressos pela média, utilizando o colorímetro digital modelo CR 300 (Minolta, New Jersey, USA), após sua calibração em uma cerâmica branca. A cor foi determinada de acordo com o Sistema CIE L* $\mathrm{a}^{*} \mathrm{~b}$ (Commision
Internationale L'Eclairage) em que os parâmetros $\mathrm{L}^{*}$ (luminosidade variando de 0 a 100), a* (-a* a a* indicando verde a vermelho) e $b^{*}\left(-b^{*} a b^{*}\right.$ indicando azul a amarelo) foram analisados (ALTAMIRANOFORTOUL; ROSELL, 2011). A variação da cor (E*) foi calculada pela equação (1):

$$
\mathrm{E}^{*}=\left[\left(\mathrm{L}^{*}-\mathrm{L}^{*}{ }_{\mathrm{ref}}\right)^{2}+\left(\mathrm{a}^{*}-\mathrm{a}_{\mathrm{ref}}^{*}\right)^{2}+\left(\mathrm{b}^{*}-\mathrm{b}^{*}{ }_{\mathrm{ref}}\right)^{2}\right]^{1 / 2}(1)
$$

Onde $\mathrm{L}^{*}{ }_{\text {ref }} \mathrm{a}^{*}{ }_{\text {ref }}$ e $\mathrm{b}^{*}{ }_{\text {ref }}$ são valores referentes à cerâmica branca utilizada na calibração do colorímetro digital $\left(\mathrm{L}^{*}{ }_{\mathrm{ref}}=97,47, \mathrm{a}^{*}{ }_{\text {ref }}=-0,16, \mathrm{~b}^{*}{ }_{\text {ref }}\right.$ $=2,23$ ) (AHRNÉ et al., 2007).

O perfil de textura dos pães foi realizado com o auxílio do texturômetro modelo TA-XT2i (Stable Micro Systems, Surrey, Reino Unido), equipado com probe cilíndrico de compressão, com $35 \mathrm{~mm}$ de diâmetro. Os parâmetros de textura determinados foram firmeza $(\mathrm{N})$, elasticidade, coesividade mastigabilidade $(\mathrm{N})$.

A avaliação desses parâmetros consiste em submeter às amostras a compressão e analisar a curva que relaciona força e tempo. A firmeza consiste na força necessária para realizar deformação, a elasticidade é a razão com que um material deformado volta ao seu estado não deformado após ser aplicado uma força, a coesividade é denominada como a força das ligações internas e a mastigabilidade é definida pela força necessária para desintegrar um alimento sólido até ficar pronto para ser engolido (SZCZESNIAK, 1998; CARR et al., 2006).

A análise instrumental de textura foi realizada sob as seguintes condições: velocidade pré-teste, do teste e pós-teste de $2,0 \mathrm{~mm} / \mathrm{s}, 5,0 \mathrm{~mm} / \mathrm{s}$ e $5,0 \mathrm{~mm} / \mathrm{s}$, respectivamente; com distância de $20 \mathrm{~mm}$, tipo de gatilho $20 \mathrm{~g}$ e tempo entre as duas compressões de $5 \mathrm{~s}$. Para análise desses parâmetros, as extremidades do pão francês foram removidas, resultando em um cilindro com aproximadamente $6,0 \mathrm{~cm}$ de comprimento (CARR; TADINI, 2003). 


\section{Análise sensorial}

Amostras de pão francês de cada panificadora foram submetidas ao teste de aceitação empregando uma escala hedônica de 9 pontos (JONES; PERYAM; THURSTONE, 1955). Foram realizadas 12 sessões, uma por panificadora, com a participação de 780 provadores voluntários, sendo 65 em cada sessão. Os provadores eram estudantes e funcionários da Universidade Federal da Paraíba (Brasil), sendo 66\% do gênero masculino e 34\% do gênero feminino, no qual 96,4\% estavam compreendidos na faixa etária de 18 a 29 anos e 3,9\% acima de 29 anos. Esse teste foi conduzido em cabines individuais.

Os pães foram avaliados quanto à aceitação considerando-se os atributos aparência, cor da casca, cor do miolo, crocância da casca, textura, aroma, sabor e quanto à aceitação global. Os pães foram considerados aceitos quando tiveram médias iguais ou superiores a 6,0 , equivalente ao termo hedônico "gostei ligeiramente".

Para avaliação da aparência, amostras do produto inteiro foram apresentadas, enquanto para os demais atributos os pães foram divididos ao meio. As amostras foram servidas de forma monádica, em pratos brancos descartáveis codificados com três dígitos aleatórios e à temperatura ambiente.

Os testes de aceitação foram realizados após aprovação pelo Comitê de Ética em Pesquisa do Centro de Ciências e Saúde da UFPB, com a referência 070/11.

\section{Análise estatística}

Os resultados das análises instrumentais das doze panificadoras foram submetidos à Análise de Variância (ANOVA) e teste Tukey, ao nível de significância de 5\%. No teste de aceitação, foram avaliados os escores médios e os percentuais dos três conceitos superiores ( 7,8 e 9$)$ e inferiores $(1,2$ e 3) da escala. Todos os resultados foram expressos pela média \pm desvio padrão.

\section{Resultados e Discussão}

Avaliação dos parâmetros de qualidade do pão francês por métodos instrumentais

Os resultados das análises de volume específico, umidade, pH e acidez estão expressos na Tabela 1.

Das 360 amostras de pães analisadas, 86,2\% apresentaram volume específico entre 5,0 e 8,0 $\mathrm{cm}^{3} / \mathrm{g}, 13,0 \%$ de 8,0 a $9,0 \mathrm{~cm}^{3} / \mathrm{g}$ e somente $0,8 \%$ de 4,5 a $4,9 \mathrm{~cm}^{3} / \mathrm{g}$, entretanto, as médias das amostras variaram de 5,72 a $8,08 \mathrm{~cm}^{3} / \mathrm{g}$ (Tabela 1) estando todas situadas no intervalo entre $5,0 \mathrm{e}$ $8,0 \mathrm{~cm}^{3} / \mathrm{g}$, considerado satisfatório (FERREIRA; OLIVEIRA; PRETTO, 2001). Geralmente, volume específico acima de, 5,0 é observado em pão francês adicionado de melhorador (KAJISHIMA; PUMAR; GERMANI, 2003; LOPES et al., 2007; CARR et al., 2006), enquanto a falta deste aditivo, o uso de massa congelada e de farinha mista promovem efeito contrário, resultando em pão com baixo volume específico $(<5,0)$ (CARR; TADINI, 2003; PEREIRA et al., 2009; BORGES et al., 2011), condição observada somente em três amostras (10\%) da panificadora L.

Para umidade dos pães foi verificada variação de $18,9 \%$ a $31,7 \%$, estando às médias situadas entre $22,21 \%$ e $28,76 \%$ (Tabela 1), com predominância de valores abaixo de $26 \%$ em oito panificadoras, indicativos de baixa umidade, condição não observada somente nas amostras da panificadora L, que obtiveram valores de umidade entre 26-31\%. $\mathrm{O}$ teor de umidade comumente observado no pão francês situa-se em torno de 30\% (FERREIRA, OLIVEIRA; PRETTO, 2001; OLIVEIRA; PIROZI; BORGES, 2007; BORGES et al., 2011), entretanto, valores mais baixos têm sido citados por outros autores. Esteller e Lannes (2005), ao avaliarem a umidade do pão francês comercializado no município de São Paulo (Brasil), obtiveram média de 26\%. Já Castro et al. (2009) verificaram neste tipo de pão, elaborado em panificadoras do município de Marabá (Brasil), umidade variando de 18,2\% a 40,1\%. Nas tabelas de composição de alimentos 
da Universidade de São Paulo - USP (2008) e do Agricultural Research Service - USDA (2012) são citados os valores $20,8 \%$ e $27,8 \%$, respectivamente.

$\mathrm{O}$ pH do pão francês variou de 4,0 a 6,2, tendo somente as amostras de quatro panificadoras apresentado todos os valores na faixa de 5,4 a 6,1 , intervalo considerado adequado para este tipo de pão (OURA; SOUMALAINEN; VISKARI, 1982), entretanto, considerando as médias das amostras (Tabela 1), cinco panificadoras apresentaram valores dentro da faixa recomendada. Apesar do pH satisfatório, essas amostras apresentaram acidez elevada variando de 4,28 a 6,11 $\mathrm{mL}$ de $\mathrm{NaOH}$ 0,1N/10 g pão, estando acima do observado (1,53,3 mL de $\mathrm{NaOH}$ 0,1N/10 g pão) em pão francês adicionado somente de leveduras (QUÍLEZ; RUIZ; ROMERO, 2006; BELZ et al., 2012). Nas sete panificadoras que produziram pães com $\mathrm{pH}$ médio abaixo de 5,4, os valores médios de acidez variaram de 4,85 a $6,40 \mathrm{~mL}$ de $\mathrm{NaOH}$ 0,1N/10 g pão.

Alguns fatores como a presença de bactérias láticas endógenas na farinha de trigo, quantidade de levedura, tipo de bactérias adicionadas à formulação, tempo e temperatura de fermentação, além do(s) tipo(s) de ácido(s) orgânico(s) presente(s), podem influenciar o $\mathrm{pH}$ e acidez dos pães (PLESSAS et al., 2011; ROBERT et al., 2006).

Os resultados das análises de cor da casca e do miolo estão expressos na Tabela 2.

Tabela 1. Volume específico, umidade, $\mathrm{pH}$ e acidez do pão francês nas doze panificadoras avaliadas.

\begin{tabular}{|c|c|c|c|c|}
\hline Panificadoras & $\begin{array}{l}\text { Volume Específico } \\
\left(\mathrm{cm}^{3} / \mathrm{g}\right)\end{array}$ & Umidade (\%) & $\mathrm{pH}$ & $\begin{array}{l}\text { Acidez }(\mathrm{mL} \text { de } \mathrm{NaOH} \\
0,1 \mathrm{~N} / 10 \mathrm{~g} \text { pão })\end{array}$ \\
\hline $\mathrm{A}$ & $7,28^{\mathrm{bc}} \pm 0,46$ & $25,29^{\mathrm{de}} \pm 1,60$ & $5,74^{\mathrm{ab}} \pm 0,08$ & $4,67^{\mathrm{cd}} \pm 0,36$ \\
\hline B & $6,46^{e} \pm 0,50$ & $26,75^{\mathrm{bc}} \pm 2,25$ & $5,86^{\mathrm{a}} \pm 0,22$ & $4,28^{\mathrm{e}} \pm 0,45$ \\
\hline $\mathrm{C}$ & $7,49^{b} \pm 0,79$ & $22,21^{\mathrm{h}} \pm 1,68$ & $5,58^{b} \pm 0,12$ & $5,44^{b} \pm 0,26$ \\
\hline $\mathrm{D}$ & $8,08^{\mathrm{a}} \pm 0,80$ & $24,93^{\mathrm{defh}} \pm 2,09$ & $5,69^{\mathrm{e}} \pm 0,28$ & $6,11^{\mathrm{a}} \pm 0,36$ \\
\hline $\mathrm{E}$ & $6,64^{\mathrm{de}} \pm 0,76$ & $23,48^{\mathrm{gh}} \pm 1,92$ & $4,69^{\mathrm{e}} \pm 0,18$ & $5,72^{b} \pm 0,73$ \\
\hline $\mathrm{F}$ & $7,47^{b} \pm 0,55$ & $24,09^{\mathrm{efg}} \pm 2,07$ & $4,70^{\mathrm{e}} \pm 0,21$ & $5,72^{b} \pm 0,64$ \\
\hline G & $7,42^{\mathrm{b}} \pm 0,82$ & $26,25^{\mathrm{bcd}} \pm 1,61$ & $4,84^{\mathrm{cde}} \pm 0,16$ & $6,40^{\mathrm{b}} \pm 0,74$ \\
\hline $\mathrm{H}$ & $7,05^{\text {bcd }} \pm 0,57$ & $27,22^{\mathrm{ab}} \pm 1,81$ & $4,78^{\mathrm{de}} \pm 0,21$ & $5,60^{\mathrm{b}} \pm 0,74$ \\
\hline I & $6,52^{\mathrm{de}} \pm 0,54$ & $23,80^{\mathrm{fgh}} \pm 1,94$ & $4,86^{\mathrm{de}} \pm 0,17$ & $5,55^{b} \pm 0,31$ \\
\hline $\mathrm{J}$ & $6,71^{\mathrm{cde}} \pm 0,75$ & $25,20^{\mathrm{def}} \pm 2,17$ & $5,05^{\mathrm{c}} \pm 0,27$ & $4,85^{\mathrm{c}} \pm 0,46$ \\
\hline $\mathrm{L}$ & $5,72^{\mathrm{f}} \pm 0,65$ & $28,76^{\mathrm{a}} \pm 1,67$ & $5,74^{\mathrm{a}} \pm 0,28$ & $4,43^{\mathrm{de}} \pm 0,37$ \\
\hline M & $6,66^{\mathrm{de}} \pm 0,33$ & $25,53^{\mathrm{cde}} \pm 1,12$ & $4,90^{\mathrm{cd}} \pm 0,27$ & $5,45^{b} \pm 0,38$ \\
\hline
\end{tabular}

Média \pm desvio padrão das análises realizadas em triplicata com 10 repetições para cada panificadora. *Médias seguidas por letras iguais, não diferem estatisticamente ao nível de 5\% de significância.

Fonte: Elaboração dos autores. 
Tabela 2. Parâmetros de cor da casca e do miolo do pão francês nas doze panificadoras avaliadas.

\begin{tabular}{|c|c|c|c|c|c|c|c|}
\hline \multirow{2}{*}{$\begin{array}{l}\text { Panifi- } \\
\text { cadoras }\end{array}$} & \multicolumn{4}{|c|}{ Cor da casca } & \multicolumn{3}{|c|}{ Cor do miolo } \\
\hline & $\mathbf{L}^{*}$ & $a^{*}$ & $\mathbf{b}^{*}$ & $\mathbf{E}^{*}$ & $\mathbf{L}^{*}$ & $a^{*}$ & $\mathbf{b}^{*}$ \\
\hline A & $71,45^{\mathrm{ab}} \pm 4,27$ & $5,86^{\text {bcdef }^{\prime}} \pm 3,00$ & $39,23^{\mathrm{abc}} \pm 4,78$ & $45,83^{\text {bcd }} \pm 4,67$ & $81,34^{\text {bcd }} \pm 1,82$ & $-1,86^{\mathrm{b}} \pm 0,14$ & $14,09^{\mathrm{abc}} \pm 0,82$ \\
\hline B & $70,71^{\mathrm{ab}} \pm 3,69$ & $3,03^{\mathrm{g}} \pm 2,84$ & $32,04^{\mathrm{bcde}} \pm 3,26$ & $40,49^{\mathrm{d}} \pm 5,87$ & $82,17^{\mathrm{bc}} \pm 2,91$ & $-1,66^{\text {bcd }} \pm 0,12$ & $14,64^{\mathrm{abc}} \pm 1,64$ \\
\hline $\mathrm{C}$ & $72,35^{\mathrm{a}} \pm 4,35$ & $4,55^{\operatorname{defg}_{ \pm}} 3,44$ & $35,06^{\mathrm{de}} \pm 6,78$ & $45,37^{\mathrm{bcd}} \pm 7,78$ & $80,61^{\mathrm{cde}} \pm 3,22$ & $-1,81^{a b} \pm 1,68$ & $15,74^{a} \pm 6,23$ \\
\hline $\mathrm{D}$ & $71,57^{\mathrm{ab}} \pm 2,95$ & $4,05^{\mathrm{fg}} \pm 2,61$ & $32,92^{\mathrm{de}} \pm 3,26$ & $50,40^{\mathrm{ab}} \pm 6,67$ & $81,00^{\text {bcde }} \pm 4,35$ & $-1,77^{b c} \pm 0,29$ & $13,57^{\mathrm{c}} \pm 0,72$ \\
\hline $\mathrm{E}$ & $70,42^{\mathrm{ab}} \pm 2,54$ & $3,49^{\mathrm{fg}} \pm 1,77$ & $33,02^{\mathrm{de}} \pm 2,65$ & $49,42^{\mathrm{ab}} \pm 4,03$ & $78,92^{\mathrm{e}} \pm 2,22$ & $-1,71^{\mathrm{bc}} \pm 0,26$ & $15,50^{\mathrm{ab}} \pm 0,92$ \\
\hline $\mathrm{F}$ & $66,48^{c} \pm 3,43$ & $7,33^{\mathrm{abc}} \pm 2,72$ & $39,68^{\mathrm{abc}} \pm 2,76$ & $41,59^{\mathrm{abc}} \pm 5,40$ & $80,53^{\text {cde }} \pm 3,64$ & $-1,28^{\mathrm{ef}^{\mathrm{e}}} \pm 0,48$ & $13,57^{\mathrm{c}} \pm 1,37$ \\
\hline G & $67,94^{\mathrm{bc}} \pm 4,14$ & $6,52^{\text {abcde }} \pm 2,93$ & $37,48^{\mathrm{bcd}} \pm 3,55$ & $44,87^{\mathrm{bcd}} \pm 8,60$ & $81,28^{\mathrm{bcde}} \pm 2,24$ & $-1,23^{\mathrm{ef}} \pm 0,13$ & $14,32^{\mathrm{abc}} \pm 1,50$ \\
\hline $\mathrm{H}$ & $66,43^{\mathrm{c}} \pm 5,68$ & $8,52^{\mathrm{a}} \pm 5,65$ & $40,60^{\mathrm{ab}} \pm 4,17$ & $41,47^{\mathrm{ab}} \pm 5,60$ & $81,57^{\mathrm{bcd}} \pm 2,56$ & $-1,40^{\operatorname{def}} \pm 0,38$ & $13,52^{\mathrm{c}} \pm 1,29$ \\
\hline I & $68,30^{\mathrm{bc}} \pm 6,48$ & $5,46^{\text {cdefg }_{1}} \pm 4,54$ & $35,47^{\text {cde }} \pm 6,20$ & $49,84^{\mathrm{ab}} \pm 5,73$ & $84,52^{a} \pm 1,28$ & $-2,17^{\mathrm{a}} \pm 0,33$ & $13,25^{\mathrm{c}} \pm 0,73$ \\
\hline $\mathrm{J}$ & $68,07^{\mathrm{bc}} \pm 4,85$ & $5,31^{\text {cdefg }_{1}} \pm 2,38$ & $43,37^{a} \pm 14,19$ & $41,77^{a} \pm 12,21$ & $81,21^{\text {bcde }} \pm 2,04$ & $-1,80^{b} \pm 0,28$ & $15,37^{\mathrm{ab}} \pm 0,72$ \\
\hline $\mathrm{L}$ & $65,72^{\mathrm{c}} \pm 5,57$ & $7,06^{\text {abcd }} \pm 4,18$ & $36,09^{\text {bcde }} \pm 4,13$ & $40,66^{\mathrm{d}} \pm 4,20$ & $79,19^{\mathrm{de}} \pm 4,32$ & $-1,13^{\mathrm{f}} \pm 0,30$ & $13,97^{\mathrm{bc}} \pm 1,66$ \\
\hline M & $65,74^{\mathrm{c}} \pm 4,80$ & $8,02^{\mathrm{ab}} \pm 3,43$ & $39,38^{\mathrm{abc}} \pm 3,49$ & $41,38^{\text {cd }} \pm 2,84$ & $83,40^{\mathrm{ab}} \pm 1,42$ & $-1,59^{\text {cde }} \pm 0,11$ & $14,12^{\mathrm{abc}} \pm 0,97$ \\
\hline
\end{tabular}

Média \pm desvio padrão das análises realizadas em triplicata com 10 repetições para cada panificadora.

*Médias seguidas por letras iguais, não diferem estatisticamente ao nível de $5 \%$ de significância.

Fonte: Elaboração dos autores.

A luminosidade da casca do pão francês variou de 50,6 a 81,4, com predominância de valores na faixa de 60 a 78, intervalo considerado satisfatório (PURLIS; SALVADORI, 2009). Luminosidade adequada foi observada em pelo menos $83,3 \%$ das amostras de todas as panificadoras, enquanto os valores médios variaram de 65,72 a 72,35 (Tabela 2). Segundo Purlis (2011), pães com luminosidade em torno de 70 apresentam boa aceitação sensorial. Entretanto, valores abaixo de 60 resultam em escurecimento excessivo e acima de 78, em coloração muito clara, indicativa de cozimento insuficiente. Com base nestes critérios, seis panificadoras (50\%) produziram pães com escurecimento excessivo, em um número máximo de amostras de 16,7\%, e somente duas (C e J) com valores acima de 78, situação observada, no máximo, em 13,3\% das amostras avaliadas. O melhor controle do tempo e temperatura de cozimento pode contribuir para minimizar a variabilidade da cor do pão, sendo recomendadas algumas condições de cozimento, como $220{ }^{\circ} \mathrm{C}$ por 10 minutos ou $200{ }^{\circ} \mathrm{C}$ durante 20 minutos, para que a luminosidade da casca do pão tipo francês fique próximo a 70 (PURLIS; SALVADORI, 2009).
Borges et al. (2011) obtiveram pães tipo francês com luminosidade da casca acima dos resultados encontrados nesta pesquisa $(82,57 \pm 6,48)$, enquanto Esteller e Lannes (2005) verificaram valor médio de 65,31 em pães obtidos em diferentes panificadoras.

Outra forma de avaliar a cor da casca dos pães é por meio do parâmetro variação de cor $\left(E^{*}\right)$, que nessa pesquisa variou de 23,3 a 82,1 , com predominância de valores abaixo de 60 , enquanto as médias variaram de 40,49 a 50,40 (Tabela 2). Segundo Ahrné et al. (2007), pães com E* acima de 60 , não apresentam boa aceitação sensorial, condição observada somente em $16,7 \%$ das amostras da panificadora $\mathrm{J}$.

Para a casca do pão, o valor de $\mathrm{a}^{*}$ variou de $-2,0$ a 14,9 e b* de 14,3 a 78,4, sendo observados valores abaixo de 8,6 para a*, em pelo menos $66,7 \%$ das amostras de dez panificadoras. Em relação ao parâmetro $b^{*}$, nove panificadoras tiveram predominância (acima de 60\%) de pães com valores acima de 34,7. Com relação às médias das amostras, foi verificado que a* variou de 3,03 a 8,52 e b* de 32,04 a 43,37 (Tabela 2). Esteller e Lannes (2005), ao avaliarem a casca do pão tipo francês, obtiveram os valores médios de 8,6 e 25,5 
para $\mathrm{a}^{*} \mathrm{e} \mathrm{b*}$, respectivamente. Altamirano-Fortoul e Rosell (2011) verificaram médias para $a^{*}$ e b*, no pão tipo baguete comercializado na Espanha, de 14,8 e 34,7 , respectivamente. Com relação a essas características, não foi verificado na literatura nenhum relato sobre valores recomendados em pão francês ou estudos que correlacionem valores desses parâmetros com análise sensorial.

O miolo do pão teve luminosidade variando de 68,1 a 86,8, com predominância de valores acima de 77 , em todas as panificadoras, enquanto os valores médios variaram de 78,92 a 84,52 (Tabela 2). Nabeshima et al. (2005) verificaram que pães com valores de luminosidade entre 70 e 77 , tiveram boa aceitação sensorial. Esteller e Lannes (2005) obtiveram valor médio abaixo do observado nessa pesquisa $(63,25 \pm 3,23)$ do miolo do pão francês. Alguns fatores como tipo de farinha e proporção dos ingredientes utilizados, podem influenciar na cor do miolo (GALLAGHER; GORMLEY; ARENDT, 2003; SALGADO et al., 2011).

Para o miolo, foi observado que $a^{*}$ variou

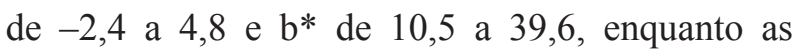
médias variaram de $-1,13$ a $-2,17$ e de 13,25 a 15,74, respectivamente. Esteller e Lannes (2005) obtiveram médias para a* e b* de $-0,4 \pm 0,68$ e 6,36 $\pm 0,68$, respectivamente.

Os resultados do perfil de textura das amostras das doze panificadoras estão expressos na Tabela 3 .

Tabela 3. Perfil de textura do pão tipo francês nas doze panificadoras avaliadas.

\begin{tabular}{|c|c|c|c|c|}
\hline \multirow{2}{*}{ Panificadoras } & \multicolumn{4}{|c|}{ TPA } \\
\hline & Firmeza (N) & Elasticidade & Coesividade & Mastigabilidade (N) \\
\hline A & $4,94^{\mathrm{abc}} \pm 2,18$ & $0,92^{b} \pm 0,02$ & $0,72^{\mathrm{a}} \pm 0,04$ & $3,18^{\mathrm{bcd}} \pm 1,14$ \\
\hline B & $3,35^{\mathrm{de}} \pm 0,96$ & $0,94^{b} \pm 0,02$ & $0,86^{\mathrm{a}} \pm 0,03$ & $2,29^{\mathrm{cd}} \pm 0,52$ \\
\hline $\mathrm{C}$ & $6,20^{\mathrm{a}} \pm 2,19$ & $0,91^{\mathrm{b}} \pm 0,02$ & $0,78^{\mathrm{a}} \pm 0,16$ & $4,07^{b} \pm 1,80$ \\
\hline $\mathrm{D}$ & $2,87^{\mathrm{e}} \pm 0,78$ & $0,93^{b} \pm 0,01$ & $0,74^{\mathrm{a}} \pm 0,02$ & $1,91^{\mathrm{d}} \pm 0,46$ \\
\hline $\mathrm{E}$ & $6,32^{\mathrm{a}} \pm 2,57$ & $0,92^{b} \pm 0,01$ & $0,72^{\mathrm{a}} \pm 0,03$ & $4,14^{\mathrm{ab}} \pm 1,40$ \\
\hline $\mathrm{F}$ & $6,13^{a} \pm 2,88$ & $0,93^{b} \pm 0,02$ & $0,75^{\mathrm{a}} \pm 0,05$ & $3,80^{\mathrm{bc}} \pm 1,58$ \\
\hline G & $4,62^{\text {bcd }} \pm 1,36$ & $0,91^{b} \pm 0,02$ & $0,75^{\mathrm{a}} \pm 0,03$ & $3,11^{\mathrm{bcd}} \pm 0,80$ \\
\hline $\mathrm{H}$ & $4,55^{\text {bcd }} \pm 1,69$ & $0,93^{b} \pm 0,02$ & $0,75^{\mathrm{a}} \pm 0,02$ & $3,01^{\text {bcd }} \pm 0,94$ \\
\hline I & $5,65^{\mathrm{ab}} \pm 1,99$ & $0,91^{b} \pm 0,03$ & $0,73^{\mathrm{a}} \pm 0,04$ & $3,70^{\mathrm{bc}} \pm 1,11$ \\
\hline $\mathrm{J}$ & $4,13^{\text {cde }} \pm 1,10$ & $1,93^{\mathrm{a}} \pm 0,03$ & $0,75^{\mathrm{a}} \pm 0,02$ & $5,71^{\mathrm{a}} \pm 5,44$ \\
\hline $\mathrm{L}$ & $3,87^{\text {cde }} \pm 1,35$ & $0,92^{b} \pm 0,02$ & $0,75^{\mathrm{a}} \pm 0,03$ & $2,63^{\mathrm{bcd}} \pm 0,76$ \\
\hline M & $4,68^{\mathrm{bcd}} \pm 1,51$ & $0,92^{b} \pm 0,01$ & $0,73^{\mathrm{a}} \pm 0,04$ & $3,04^{\text {bcd }} \pm 0,90$ \\
\hline
\end{tabular}

Média \pm desvio padrão das análises realizadas em triplicata com 10 repetições para cada panificadora.

*Médias seguidas por letras iguais, não diferem estatisticamente ao nível de $5 \%$ de significância.

Fonte: Elaboração dos autores.

A firmeza do pão francês variou de $1,94 \mathrm{~N}$ a 13,3 $\mathrm{N}$, tendo ocorrido valores de até $8,5 \mathrm{~N}$ em $94,2 \%$ das amostras, enquanto as médias variaram de 2,87 $\mathrm{N}$ a $6,32 \mathrm{~N}$. Na literatura, foi observado que valores de firmeza entre $0,8 \mathrm{~N}$ e 8,5 $\mathrm{N}$ resultavam em pães com boa aceitação sensorial (SILVA; YONAMINE; MITSUIKI, 2003; CARR et al., 2006; OLIVEIRA; PIROZI; BORGES, 2007). Borges et al. (2011), ao adicionarem $10 \%$ de farinha de linhaça à formulação do pão francês, verificaram aumento de firmeza de 1,40 N para 7,32 N. Carr et al. (2006) verificaram em pão francês, adicionado da enzima alfa-amilase e emulsificantes, firmeza média de $6,24 \mathrm{~N}$, e quando o mesmo produto foi submetido ao congelamento por cinco dias, a média aumentou para 7,54 N. 
Alguns fatores como incorporação adicional de glúten, utilização de massa ácida, retrogradação das amilopectinas, elaboração de pães com massa congelada, adição de inulina e sal tendem a aumentar a firmeza do pão, no entanto, o uso de alguns tipos de aditivos, como o ácido ascórbico e enzima alfa amilase, retarda o endurecimento (CARR; TADINI, 2003; SILVA; YONAMINE; MITSUIKI, 2003; MANDALA, 2005; ALTAMIRANO-FOURTOUL; ROSELL, 2011).

Em relação aos demais parâmetros de textura do pão francês, foi verificado que a média da mastigabilidade variou de $1,91 \mathrm{~N}-5,71 \mathrm{~N}$ e da elasticidade de $0,91-1,93$. Com relação à coesividade, foram observados nessa pesquisa valores médios entre 0,72 e 0,86, enquanto Carr e Tadini (2003) obtiveram valores entre 0,65 0,72 . As amostras da panificadora B foram as que apresentaram maior coesividade, enquanto as da panificadora $\mathrm{J}$ maior elasticidade e mastigabilidade. Considerando que ambas apresentaram os escores médios mais baixos no atributo crocância, é possível que haja relação entre essas características e esse atributo, o que deve ser investigado.

\section{Avaliação sensorial}

Das doze panificadoras avaliadas, seis tiveram suas amostras aceitas em todos os atributos (Tabela 4), destacando-se duas destas por não apresentarem nenhum escore médio abaixo de 7,0 ( $\mathrm{C}$ e $\mathrm{M})$, enquanto as panificadoras $\mathrm{E}$ e $\mathrm{J}$, apresentaram o maior número de atributos que não alcançaram esse valor (6 e 7, respectivamente).

As seis panificadoras com amostras rejeitadas apresentaram escore médio abaixo de 6,0 no atributo crocância, tendo somente uma $(\mathrm{J})$ sido rejeitada também nos atributos aparência, aroma e sabor, estando com maior necessidade de adequação. Esses resultados indicam que a falta de crocância foi o principal problema de qualidade detectado nos pães.

Carr et al. (2006), ao avaliarem o pão tipo francês comercial e o obtido a partir de massa congelada, quanto aos atributos brilho, rugosidade e crocância da casca, aspecto da pestana, firmeza do miolo e sabor, verificaram que os pães não diferiram somente quanto a crocância da casca, obtendo escores médio de 6,8 e 6,5, respectivamente. Para os demais atributos, o pão de massa congelada teve maior aceitação, com médias acima de 6,7.

Tabela 4. Aceitação média por atributos e aceitação global do pão tipo francês comercializado nas doze panificadoras.

\begin{tabular}{lcccccccc}
\hline \multirow{2}{*}{$\begin{array}{l}\text { Panifi- } \\
\text { cadoras }\end{array}$} & Aparência & $\begin{array}{c}\text { Cor da } \\
\text { casca }\end{array}$ & $\begin{array}{c}\text { Cor do } \\
\text { miolo }\end{array}$ & $\begin{array}{c}\text { Crocância } \\
\text { da casca }\end{array}$ & Aroma & Sabor & Textura & $\begin{array}{c}\text { Aceitação } \\
\text { global }\end{array}$ \\
\hline A & $8,0 \pm 1,2$ & $8,0 \pm 1,1$ & $7,7 \pm 1,0$ & $6,0 \pm 1,7$ & $7,3 \pm 1,5$ & $7,4 \pm 1,3$ & $7,9 \pm 1,0$ & $7,3 \pm 1,1$ \\
B & $7,2 \pm 1,3$ & $7,7 \pm 1,8$ & $7,2 \pm 1,4$ & $5,3 \pm 2,1$ & $7,0 \pm 1,6$ & $7,5 \pm 1,2$ & $7,6 \pm 1,4$ & $7,1 \pm 1,2$ \\
C & $7,3 \pm 1,5$ & $7,4 \pm 1,4$ & $7,6 \pm 1,7$ & $7,0 \pm 1,7$ & $7,0 \pm 1,7$ & $7,4 \pm 1,7$ & $7,4 \pm 1,5$ & $7,3 \pm 1,3$ \\
D & $7,0 \pm 1,6$ & $7,3 \pm 1,4$ & $7,3 \pm 1,3$ & $6,8 \pm 1,7$ & $6,8 \pm 1,4$ & $6,9 \pm 1,5$ & $6,8 \pm 1,5$ & $7,0 \pm 1,1$ \\
E & $6,4 \pm 1,5$ & $7,2 \pm 1,4$ & $6,8 \pm 1,3$ & $5,6 \pm 1,0$ & $6,4 \pm 1,6$ & $6,6 \pm 1,9$ & $7,4 \pm 1,2$ & $6,6 \pm 1,3$ \\
F & $7,4 \pm 1,3$ & $7,5 \pm 1,0$ & $7,6 \pm 1,1$ & $5,2 \pm 1,7$ & $6,3 \pm 1,4$ & $6,4 \pm 1,7$ & $7,0 \pm 1,8$ & $6,9 \pm 1,2$ \\
G & $7,2 \pm 1,3$ & $7,5 \pm 1,3$ & $7,3 \pm 1,1$ & $5,9 \pm 1,7$ & $6,6 \pm 1,4$ & $6,8 \pm 1,4$ & $7,0 \pm 1,7$ & $6,8 \pm 1,2$ \\
H & $7,6 \pm 1,2$ & $7,8 \pm 1,3$ & $7,6 \pm 1,0$ & $5,9 \pm 1,9$ & $6,9 \pm 1,6$ & $6,8 \pm 1,6$ & $7,1 \pm 1,4$ & $7,0 \pm 1,4$ \\
I & $7,1 \pm 1,7$ & $7,6 \pm 1,2$ & $6,0 \pm 1,2$ & $6,7 \pm 1,8$ & $7,0 \pm 1,6$ & $7,3 \pm 1,6$ & $7,1 \pm 1,7$ & $7,2 \pm 1,4$ \\
J & $5,7 \pm 2,4$ & $6,9 \pm 2,2$ & $7,7 \pm 1,9$ & $5,5 \pm 2,4$ & $5,9 \pm 2,4$ & $5,8 \pm 2,3$ & $6,2 \pm 2,4$ & $6,0 \pm 2,0$ \\
L & $7,7 \pm 1,1$ & $7,9 \pm 1,1$ & $7,9 \pm 1,1$ & $6,4 \pm 2,0$ & $7,3 \pm 1,5$ & $7,7 \pm 1,4$ & $7,9 \pm 1,7$ & $7,7 \pm 1,3$ \\
M & $8,0 \pm 1,2$ & $7,8 \pm 1,3$ & $7,9 \pm 1,1$ & $7,2 \pm 1,7$ & $7,1 \pm 1,5$ & $7,2 \pm 1,4$ & $7,1 \pm 1,7$ & $7,5 \pm 1,3$ \\
\hline
\end{tabular}

Média \pm desvio padrão das análises realizadas com 65 provadores por panificadora.

Fonte: Elaboração dos autores. 
Tabela 5. Porcentagens dos 3 conceitos superiores da escala hedônica (valores 7,8 e 9) e dos 3 conceitos inferiores (valores 1,2 e 3 ) para os atributos sensoriais.

\begin{tabular}{|c|c|c|c|c|c|c|c|c|c|c|c|c|c|}
\hline & anificadoras & A & B & $\mathrm{C}$ & $\mathrm{D}$ & $\mathrm{E}$ & $\mathrm{F}$ & $\mathrm{G}$ & $\mathrm{H}$ & I & $\mathrm{J}$ & $\mathrm{L}$ & M \\
\hline \multirow{2}{*}{ Aparência } & $\begin{array}{c}\text { Proporção dos } 3 \\
\text { Conceitos Superiores }\end{array}$ & 95,4 & 87,7 & 89,2 & 80 & 75,4 & 90,8 & 89,2 & 93,8 & 81,5 & 66,2 & 93,8 & 95,4 \\
\hline & $\begin{array}{c}\text { Proporção dos } 3 \\
\text { Conceitos Inferiores }\end{array}$ & 1,5 & 3,1 & 4,6 & 12,3 & 13,8 & 4,6 & 6,2 & 3,1 & 12,3 & 26,2 & 4,6 & 3,1 \\
\hline \multirow{2}{*}{$\begin{array}{l}\text { Cor da } \\
\text { casca }\end{array}$} & $\begin{array}{c}\text { Proporção dos } 3 \\
\text { Conceitos Superiores }\end{array}$ & 95,4 & 84,6 & 89,2 & 89,2 & 81,5 & 96,9 & 90,8 & 95,4 & 90,8 & 69,2 & 96,9 & 93,8 \\
\hline & $\begin{array}{c}\text { Proporção dos } 3 \\
\text { Conceitos Inferiores }\end{array}$ & 1,5 & 9,2 & 4,6 & 3,1 & 10,8 & 0 & 6,2 & 4,6 & 1,5 & 27,7 & 1,5 & 4,6 \\
\hline \multirow{2}{*}{$\begin{array}{l}\text { Cor do } \\
\text { miolo }\end{array}$} & $\begin{array}{c}\text { Proporção dos } 3 \\
\text { Conceitos Superiores }\end{array}$ & 95,4 & 90,8 & 90,8 & 92,3 & 89,2 & 93,8 & 93,8 & 96,9 & 95,4 & 80 & 98,5 & 95,4 \\
\hline & $\begin{array}{c}\text { Proporção dos } 3 \\
\text { Conceitos Inferiores }\end{array}$ & 0 & 3,1 & 3,1 & 1,5 & 0 & 0 & 0 & 1,5 & 4,6 & 13,8 & 1,5 & 1,5 \\
\hline \multirow{2}{*}{$\begin{array}{l}\text { Crocância } \\
\text { da casca }\end{array}$} & $\begin{array}{c}\text { Proporção dos } 3 \\
\text { Conceitos Superiores }\end{array}$ & 66,2 & 50,8 & 84,6 & 81,5 & 69,2 & 52,3 & 66,2 & 63,1 & 78,5 & 58,5 & 72,3 & 87,7 \\
\hline & $\begin{array}{c}\text { Proporção dos } 3 \\
\text { Conceitos Inferiores }\end{array}$ & 21,5 & 35,4 & 12,3 & 13,8 & 24,6 & 33,8 & 21,5 & 24,6 & 13,8 & 32,3 & 16,9 & 7,7 \\
\hline \multirow{2}{*}{ Aroma } & $\begin{array}{c}\text { Proporção dos } 3 \\
\text { Conceitos Superiores }\end{array}$ & 84,6 & 83,1 & 90,8 & 86,2 & 76,9 & 73,8 & 73,8 & 75,4 & 81,5 & 66,2 & 92,3 & 87,7 \\
\hline & $\begin{array}{c}\text { Proporção dos } 3 \\
\text { Conceitos Inferiores }\end{array}$ & 6,2 & 4,6 & 4,6 & 7,7 & 15,4 & 12,3 & 4,6 & 9,2 & 7,7 & 18,5 & 4,6 & 4,6 \\
\hline \multirow{2}{*}{ Sabor } & $\begin{array}{c}\text { Proporção dos } 3 \\
\text { Conceitos Superiores }\end{array}$ & 93,8 & 95,4 & 84,6 & 83,1 & 75,4 & 78,5 & 86,2 & 83,1 & 86,2 & 63,1 & 92,3 & 84,6 \\
\hline & $\begin{array}{c}\text { Proporção dos } 3 \\
\text { Conceitos Inferiores }\end{array}$ & 3,1 & 1,5 & 9,2 & 7,7 & 16,9 & 15,4 & 6,2 & 15,4 & 10,8 & 27,7 & 3,1 & 1,5 \\
\hline \multirow[t]{2}{*}{ Textura } & $\begin{array}{c}\text { Proporção dos } 3 \\
\text { Conceitos Superiores }\end{array}$ & 96,9 & 93,8 & 89,2 & 83,1 & 92,3 & 83,1 & 83,1 & 90,8 & 87,7 & 69,2 & 93,8 & 86,2 \\
\hline & $\begin{array}{c}\text { Proporção dos } 3 \\
\text { Conceitos Inferiores }\end{array}$ & 0 & 6,2 & 9,2 & 10,8 & 3,1 & 12,3 & 7,7 & 6,2 & 9,2 & 21,5 & 0 & 10,8 \\
\hline \multirow{2}{*}{$\begin{array}{c}\text { Aceitação } \\
\text { global }\end{array}$} & $\begin{array}{c}\text { Proporção dos } 3 \\
\text { Conceitos Superiores }\end{array}$ & 93,8 & 90,8 & 90,8 & 84,6 & 78,5 & 90,8 & 90,8 & 81,5 & 89,2 & 64,6 & 95,4 & 90,8 \\
\hline & $\begin{array}{c}\text { Proporção dos } 3 \\
\text { Conceitos Inferiores }\end{array}$ & 1,5 & 6,2 & 4,6 & 0 & 7,7 & 4,6 & 6,2 & 6,2 & 7,7 & 23,1 & 1,5 & 0 \\
\hline
\end{tabular}

Fonte: Elaboração dos autores.

Oliveira, Pirozi e Borges (2007) e Borges et al. (2011) verificaram que o pão francês incrementado com farinha de linhaça (10\% e $15 \%)$ teve maior firmeza na analise instrumental do que a amostra controle, no entanto, essa diferença não foi percebida pelos consumidores durante a análise sensorial da textura. Os pães com linhaça tiveram boa aceitação quanto aos atributos aparência, aroma, cor, textura e aceitação global, obtendo escores médios acima de 7,8 . 
A cor da casca, aparência, textura e flavor são os atributos mais utilizados para avaliar a aceitação do pão (AHRNÉ et al., 2007; ABDULLAH, 2008). Segundo Hersleth et al. (2005), a textura do pão foi considerada pelos consumidores a característica mais importante, por indicar o grau de frescor, enquanto a aparência não foi enfatizada como determinante na aceitabilidade do produto. Já Berno, Spoto e Canniatti-Brazaca (2007) observaram que os atributos aparência física e cor são determinantes na escolha do pão no momento da compra.

Quanto aos percentuais dos 3 escores superiores ( 7,8 e 9) e inferiores (1, 2 e 3$)$ da escala hedônica foram observados valores variando de 50,8-98,5\% e de $0-35,4 \%$, respectivamente. O maior percentual dos 3 escores superiores foi observado nas amostras da panificadora L, para o atributo cor do miolo, enquanto o maior percentual nos 3 escores inferiores foi obtido pelas amostras da panificadora $B$, no atributo crocância.

As amostras da panificadora A, apesar de aceitas com base nos escores médios em todos os atributos avaliados, apresentou percentual acima de $20 \%$ (Tabela 5) dos 3 escores inferiores quanto a crocância, condição observada em todas as amostras rejeitadas quanto a esse atributo. Foi observado ainda que as amostras de todas as panificadoras, com exceção da $\mathrm{M}$, apresentaram o maior percentual dos 3 escores inferiores nesse atributo, confirmando a falta de crocância como principal problema de qualidade nos pães avaliados.

Com base na análise dos percentuais dos 3 escores superiores, as amostras das panificadoras A, $\mathrm{C}$ e $\mathrm{L}$ se destacaram das demais, pois alcançaram, em pelo menos 6 atributos, percentuais em torno de $90 \%$, enquanto nas amostras da panificadora $\mathrm{J}$, nenhum atributo alcançou esse percentual, confirmando sua menor aceitação. Resultados semelhantes foram obtidos quando os percentuais dos 3 escores inferiores foram avaliados, tendo as amostras das panificadoras A, C e L apresentado o maior número de atributos com menores percentuais, não excedendo $5 \%$ em pelo menos 5 atributos. Novamente, as amostras da panificadora $\mathrm{J}$ não apresentaram nenhum atributo nessa condição.

\section{Conclusões}

Os resultados das análises instrumentais indicaram que os principais problemas de qualidade do pão tipo francês foram umidade baixa e acidez elevada, tendo o volume específico apresentado maior adequação. No teste de aceitação sensorial, a falta de crocância foi responsável pela rejeição das amostras de $50 \%$ das panificadoras, tendo somente uma destas (J) sido rejeitada também quanto à aparência, aroma e sabor, apresentando maior necessidade de adequação.

Portanto, futuros estudos devem ser realizados, correlacionando análises instrumentais e sensoriais, especialmente umidade e acidez com atributos sensoriais como textura, aroma e sabor, na tentativa de estabelecer limites mínimos e máximos aceitáveis para essas características.

\section{Referências}

AMERICAN ASSOCIATION OF CEREAL CHEMISTS -AACC. Approved methods of the AACC. $8^{\text {th }}$ ed. St. Paul, MN: Method n 10- 11, 2000.

ABDULLAH, M. Z. Quality evaluation of bakery products - computer vision technology for food quality evaluation. Burlington, EUA: D.-W. Sun, 2008.

AGRICULTURAL RESEARCH SERVICE - USDA. Banco de dados de nutrientes nacional de referência padrão. 2012. Disponível em: http://ndb.nal.usda.gov/ ndb/foods/show/5423. Acesso em: 05 jan. 2013.

AHRNÉ, A.; ANDERSSON, C. G.; FLOBERG, P.; ROSÉN, J.; LINGNERT, H. Effect of crust temperature and water content on acrylamide formation during baking of white bread: Steam and falling temperature baking. Swiss Society of Food Science and Technology, Göteborg, v. 40, n. 10, p. 1708-1715, 2007.

ALTAMIRANO-FORTOUL, R.; ROSELL, C. M. Physico-chemical changes in breads from bake off technologies during storage. Food Science and Technology, Valencia, v. 44, n. 3, p. 631-636, 2011. 
ASSOCIATION OF OFFICIAL ANALYTICAL CHEMISTS - AOAC. Official methods of analysis of AOAC International. 16. ed. Gaitheersburg: AOAC, 1997.

BÁRCENAS, M. E.; O-KELLER, J. D.; ROSELL, C. M. Influence of different hydrocolloids on major wheat dough components (gluten and starch). Journal of Food Engineering, Amsterdam, v. 94, n. 3-4, p. 241-247, 2009.

BELZ, M. C. E.; MAIRINGER, R.; ZANNINI, E.; RYAN, L. A. M.; CASHMAN, K. D.; ARENDT, E. $\mathrm{K}$. The effect of sourdough and calcium propionate on the microbial shelf-life of salt reduced bread. Applied Microbiology and Biotechnology, Berlin, v. 96, n. 2, p. 493-501, 2012.

BERNO, L. I.; SPOTO, M. H. F.; CANNIATTIBRAZACA, S. G. Avaliação química e aceitabilidade de pão enriquecido com proteína concentrada do soro de leite bovino (whey protein). Alimentros e Nutrição, Araraquara, v. 18, n. 1, p. 41-49, 2007.

BORGES, J. T. da S.; PIROZI, M. R.; PAULA, C. D. de; RAMOS, D. L.; CHAVES, J. B. P. Caracterização físico-química e sensorial de pão de sal enriquecido com farinha integral de linhaça. Boletim do CEPPA, Curitiba, v. 29, n. 1, p. 83-96, 2011.

CALLEJO, M. J. Present situation in the descriptive sensory analysis of bread. Journal of Sensory Studies, New York, v. 26, n. 4, p. 255-268, 2011.

CARR, L. G.; RODAS, M. A. B.; DELLA TORRE, J. C. M.; TADINI, C. C. Physical, textural and sensory characteristics of 7-day frozen part-baked French bread. Lebensmittel - Wissenschaft und - Technologie, New York, v. 39, n. 5, p. 540-547, 2006.

CARR, L. G.; TADINI, C. C. Influence of yeast and vegetable shortening on physical and texture parameters of frozen part baked French bread. Lebensmittel Wissenschaft und - Technologie, New York, v. 36, n. 6, p. 609-614, 2003.

CASTRO, E. M.; DANTAS, A. A. S.; DA CUNHA, M. A. E.; SANTOS, E. R. Evaluation of the quality of french bread manufactured in the city of Marabá, PA. Higiene Alimentar, São Paulo, v. 23, n. 168-69, p. 65-71, 2009.

CURIC, D.; NOVOTNI, D.; SKEVIN, D.; ROSELL, C. M.; COLLAR, C.; LE BAIL, COLIC-BARIC, I.; GABRIC, D. Design of a quality index for the objective evaluation of bread quality: application of wheat breads using selected bake off technology for bread making. Food Research Internacional, Amsterdam, v. 41, n. 7, p. 714-719, 2008.
ESTELLER, M. S.; LANNES, S. C. da S. Parâmetros complementares para fixação de identidade e qualidade de produtos panificados. Ciência e Tecnologia de Alimentos, Campinas, v. 25, n. 4, p. 802-806, 2005.

FERREIRA, S. M. R.; OLIVEIRA, P. V.; PRETTO, D. Parâmetros de qualidade do pão francês. Boletim do CEPPA, Curitiba, v. 19, n. 2, p. 301-318, 2001.

GALLAGHER, E.; GORMLEY, T. R.; ARENDT, E. K. Crust and crumb characteristics of gluten free breads. Journal of Food Engineering, Amsterdam, v. 56, n. 2-3, p. 153-61, 2003.

GIANNOU, V.; KESSOGLOU, V; TZIA, C. Quality and safety characteristics of bread made from frozen dough. Trends in Food Science \& Technology, Amsterdam, v. 14, n. 3, p. 99-108, 2003.

HAYAKAWA, E.; UKAI, N.; NISHIDA, J.; KAZAMI, Y.; KOHYAMA, K. Lexicon for the sensory quality description of French bread in Japan. Journal of Sensory Studies, Indianapolis, v. 25, n. 1, p. 76-93, 2010.

HERSLETH, M.; BERGGREN, R.; WESTAD, F.; MARTENS, M. Perception of bread: a comparison of consumers and trained assessors. Journal of Food Science, Chicago, v. 70, n. 2, p. S95-S101, 2005.

INSTITUTO BRASILEIRO DE GEOGRAFIA E ESTATÍSTICA - IBGE. Brasileiro come menos arroz com feijão e mais comida industrializada em casa. 2010. Disponível em: <http://www.ibge.com.br/ home/presidencia/noticias/noticia_visualiza.php?id noticia $=1788 \&$ \&id_pagina=1>. Acesso em: 05 maio 2012.

JONES, L. V.; PERYAM, D. R.; THURSTONE, L. L. Development of a scale for measuring soldiers' food preferences. Journal of Food Science, Chicago, v. 20, n. 5, p. 512-520, 1955.

JOYE, I. J.; LAGRAIN, B.; DELCOUR, J. A. Use of chemical redox agents and exogenous enzymes to modify the protein network during breadmaking - a review. Journal of Cereal Science, New York, v. 50, n. 1, p. 11-21, 2009.

KAJISHIMA, S.; PUMAR, M.; GERMANI, R. Efeito de adição de diferentes sais de cálcio nas características da massa e na elaboração de pão francês. Ciência e Tecnologia de Alimentos, Campinas, v. 23, n. 2, p. 222225, 2003.

LOPES, A. S.; ORMENESE, R. C. S. C.; MONTENEGRO, F. M.; FERREIRA JÚNIOR, P. G. Influência do uso simultâneo de ácido ascórbico e azodicarbonamida na qualidade do pão francês. Ciência e Tecnologia de Alimentos, Campinas, v. 27, n. 2, p. $307-$ 312, 2007. 
MANDALA, I. G. Physical properties of fresh and frozen stored, microwave-reheated breads, containing hydrocolloids. Journal of Food Engineering, Amsterdam, v. 66, n. 3, p. 291-300, 2005.

NABESHIMA, E. H.; ORMENESE, R. de C. S. C.; MONTENEGRO, F. M.; TODA, E.; SADAHIRA, M. S. Propriedades tecnológicas e sensoriais de pães fortificados com ferro. Ciência e Tecnologia de Alimentos, Campinas, v. 25, n. 3, 506-511, 2005.

OLIVEIRA, T. M.; PIROZI, M. R.; BORGES, J. T. S. Elaboração de pão de sal utilizando farinha mista de trigo e linhaça. Alimentos e Nutrição, Araraquara, v. 18, n. 2, p. 141-150, 2007.

OURA, E.; SOUMALAINEN, H.; VISKARI, R. Breadmaking. In: ROSE, H. (Ed.). Economic microbiology. New York: Academic Press, 1982. v. 7, p. $87-146$

PEREIRA, E. P. R.; AMORIM, E. O. C.; AMBIEL H. C. I.; CHANG, Y. K. Influence of oxidizing agents on the rheological properties of doughs prepared from white flour and whole-grain flour and on the specific volume of french rolls. Brazilian Journal Food Technology, Campinas, v. 12, n. 3, p. 161-171, 2009.

PLESSAS, S.; ALEXOPOULOS, A.; MANTZOURANI, I.;KOUTINAS,A.;VOIDAROU,C.; STAVROPOULOU, E.; BEZIRTZOGLOU, E. Application of novel starter cultures for sourdough bread production. Anaerobe, London, v. 17, n. 6, p. 486-489, 2011.

PURLIS, E. Bread baking: technological considerations based on process modeling and simulation. Journal of Food Engineering, Amsterdam, v. 103, n. 1, p. 92-102, 2011.
PURLIS, E.; SALVADORI, V. O. Modelling the browning of bread during baking. Food Research International, Amsterdam, v. 42, n. 9, p. 865-870, 2009.

QUÍLEZ, J.; RUIZ, J. A.; ROMERO, M. P. Relationships between sensory flavor evaluation and volatile and nonvolatile compounds in commercial wheat bread type baguette. Journal of Food Science, Chicago, v. 71, n. 6, p. S423-S427, 2006.

ROBERT, H.; GABRIEL, V.; LEFEBVRE, D.; RABIER, P.; VAYSSIER, Y.; FONTAGNÉ-FAUCHER, C. Study of the behaviour of Lactobacillus plantarum and Leuconostoc starters during a complete wheat sourdough breadmaking process. Lebensmittel - Wissenschaft und - Technologie, New York, v. 39, n. 3 p. 256-265, 2006.

SALGADO, J. M.; RODRIGUES, B. S.; DONADOPESTANA, C. M.; DIAS, C. T. dos S.; MORZELLE M. C. Cupuassu (Theobroma grandiflorum) peel as potential source of dietary fiber and phytochemicals in wholebread preparations. Plant Foods for Human Nutritian, Netherlands, v. 66, n. 4, p. 384-390, 2011.

SILVA, M. E. M. P.; YONAMINE, G. E.; MITSUIKI, L. Development and evaluation of homemade french bread containing no salt. Brazilian Journal Food Technology, Campinas, v. 6, n. 2, p. 229-236, 2003.

SZCZESNIAK, A. S. Sensory texture profiling historical and scientific perspectives. Food Technology, Chicago, v. 52, n. 8, p. 54-57, 1998.

UNIVERSIDADE DE SÃO PAULO - USP. Tabela brasileira de composição de alimentos. TBCAUSP 5.0. 2008. Disponível em: <http://www.fcf.usp.br/tabela/ resultado.asp? IDLetter $=$ A\&IDNumber $=212>$. Acesso em: 11 jan. 2013. 